\title{
Khovanov homology, its definitions and ramifications
}

\author{
by
}

Oleg Viro (Uppsala and St. Petersburg)

\begin{abstract}
Mikhail Khovanov defined, for a diagram of an oriented classical link, a collection of groups labelled by pairs of integers. These groups were constructed as the homology groups of certain chain complexes. The Euler characteristics of these complexes are the coefficients of the Jones polynomial of the link. The original construction is overloaded with algebraic details. Most of the specialists use adaptations of it stripped off the details. The goal of this paper is to overview these adaptations and show how to switch between them. We also discuss a version of Khovanov homology for framed links and suggest a new grading for it.
\end{abstract}

1. Introduction. For a diagram $D$ of an oriented link $L$, Mikhail Khovanov [7] constructed a collection of groups $\mathcal{H}^{i, j}(D)$ such that

$$
K(L)(q)=\sum_{i, j} q^{j}(-1)^{i} \operatorname{dim}_{\mathbb{Q}}\left(\mathcal{H}^{i, j}(D) \otimes \mathbb{Q}\right),
$$

where $K(L)$ is a version of the Jones polynomial of $L$. These groups are constructed as homology groups of certain chain complexes.

Khovanov homology has proved to be a powerful and useful tool in low dimensional topology. Recently Jacob Rasmussen [16] has applied it to problems of estimating the slice genus of knots and links. He defined a knot invariant which gives a lower bound for the slice genus and which, for knots with only positive crossings, allowed him to find the exact value of the slice genus. Using this technique, he has found a new proof of the Milnor conjecture on the slice genus of toric knots. This is the first proof which does not depend on the technique of gauge theory. It is much simpler than all the previously known proofs.

Khovanov homology is not an isolated phenomenon any more. Since 1999, when Khovanov [7] discovered it, several similar link homology theories have been discovered (see [8], [10-12], [15], [3]). Khovanov homology seems to be the simplest of them.

2000 Mathematics Subject Classification: 57M25, 57M27. 
As Khovanov homology becomes one of the mainstream techniques in topology, the details of its definition and internal structure deserve a careful consideration.

The original construction of $\mathcal{H}^{i, j}(D)$ is overloaded with algebraic details. More topologist-friendly versions of this construction were presented by Dror Bar-Natan [2] and the author [18]. The constructions in [2] and [18] differ only by inessential details from each other and from the original paper by Khovanov [7]. One can easily recognize all the details of any of them in the other one.

Nonetheless, in most of the papers on Khovanov homology, the differences between [2] and [18] are taken too seriously. In this paper I discuss the constructions again. I begin with the approach of [18]. Its main idea is to show how one could invent Khovanov homology starting with the Kauffman bracket model for the Jones polynomial and a general desire to upgrade (i.e., categorify) the Jones polynomial. Then I identify this construction with the construction of [2] and [7].

The Khovanov homology is closer to the Kauffman bracket, which is an invariant of non-oriented, but framed links, than to the Jones polynomial, which is an invariant of oriented, but non-framed links. The corresponding modification of Khovanov homology is presented in Section 6. This allows us to write down a categorification of the Kauffman skein relation for the Kauffman bracket. The skein relation gives rise to a homology sequence.

I am grateful to Józef Przytycki for his stimulating interest in this paper and to Alexander Shumakovitch for numerous interesting discussions and valuable remarks.

\section{Preliminary exposition of link matters}

2.1. Links, their framings and orientations. Let me recall the basic notions. By a link we mean a smooth closed 1-dimensional submanifold of $\mathbb{R}^{3}$. Links $L_{0}, L_{1}$ are called (ambient) isotopic if there exists an isotopy $h_{t}: \mathbb{R}^{3} \rightarrow \mathbb{R}^{3}, t \in[0,1]$, with $h_{0}=\mathrm{id}$ and $h_{1}\left(L_{0}\right)=L_{1}$. Up to isotopy a link is characterized by its diagram, i.e., a generic projection of the link to a plane, decorated at each double point to specify over-crossing and under-crossing branches. A double point on a link diagram is called a crossing.

By a framing of a link we mean a non-vanishing normal vector field on the link. We consider framings up to isotopy. A link diagram defines a class of isotopic framings, which contains both framings annihilated by the projection and framings whose vectors are projected to non-zero vectors. The framings of the latter type are called blackboard framings for an obvious reason. 
For an oriented framed link (i.e., a link with a distinguished framing) the self-linking number is the linking number between the link and the result of a small shift of it along the framing.

The self-linking number depends on the orientation: reversing the orientation of a single component of a link changes the self-linking number by the doubled linking number of the component and its complement. In particular, in the case of a knot (a one-component link) the self-linking number does not depend on its orientation. (It depends on the orientation of the ambient space, which we will assume to be fixed.)

A framing of a knot is characterized by its self-linking number up to isotopy. Hence the isotopy class of a framing is described by the self-linking numbers of its components. Thus a framing of a link can be characterized up to isotopy by assignment of an integer to each connected component of the link. A framing of a knot with self-linking number $n$ is called an $n$-framing.

With a crossing point of a diagram of an oriented link we associate the local writhe number equal to

- +1 if at the point the diagram looks like $\lambda$, and

- -1 if it looks like $Y$.

The sum of the local writhe numbers over all crossing points of a link diagram $D$ is called the writhe number of $D$ and denoted by $w(D)$.

The writhe number of a link diagram $D$ is equal to the self-linking number of the corresponding blackboard framing.

Addition of a little kink to a diagram (i.e., the first Reidemeister move) changes the writhe number by \pm 1 . Therefore any framing of a link can be realized as a blackboard framing: for this one should add an appropriate number of kinks.

2.2. Jones polynomial and Kauffman bracket. In this paper we will deal with two polynomial link invariants closely related to each other: the Jones polynomial $V_{L}$ and the Kauffman bracket $\langle L\rangle$. The Jones polynomial is defined on the set of oriented links, the Kauffman bracket is defined on the set of framed links.

The Kauffman bracket of a framed link is a Laurent polynomial in $A$. It is defined by the following properties:

(1) Normalization. 〈unknot with 0-framing $\rangle=1$.

(2) Stabilization. $\langle L \amalg$ unknot with 0 -framing $\rangle=\left(-A^{2}-A^{-2}\right)\langle L\rangle$, where $\amalg$ stands for disjoint sum.

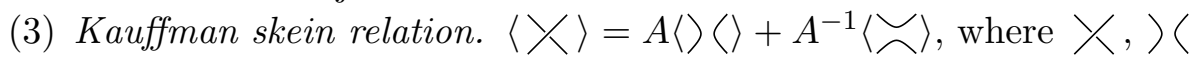
and $\asymp$ stand for links defined by their diagrams with blackboard framing which coincide outside a disk and in the disk look as their notations. 
The Jones polynomial of an oriented link is a Laurent polynomial in $t^{1 / 2}$ defined by the following properties:

(1) Normalization. $V_{\text {unknot }}=1$.

(2) Stabilization. $V_{L} \amalg$ unknot $=\left(-t^{1 / 2}-t^{-1 / 2}\right) V_{L}$, where $\amalg$ stands for disjoint sum.

(3) Jones skein relation. $t^{-1} V_{\nearrow}-t V_{\nearrow}=\left(t^{1 / 2}-t^{-1 / 2}\right) V_{\nearrow}$, where $\aleph, \chi$ and $)($ stand for oriented link diagrams coinciding outside a disk and in the disk looking as their notations.

When one speaks about the Kauffman bracket of a link diagram, this means the Kauffman bracket of the link represented by the diagram and equipped with the blackboard framing.

If the link is both oriented and framed, both the Jones polynomial and Kauffman bracket make sense and each of them can be expressed in terms of the other and the self-linking number of the framing. Namely, for an oriented link $L$ with diagram $D$,

$$
V_{L}\left(A^{-4}\right)=(-A)^{-3 w(D)}\langle D\rangle
$$

2.3. Kauffman state sum. The Kauffman skein relation applied consecutively to all crossings of a link diagram allows one to express the Kauffman bracket of the diagram as a linear combination of the Kauffman brackets of collections of circles embedded in the plane. Then the Stabilization and Normalization properties complete the calculation.

This calculation can be rearranged as follows. The summands in the Kauffman skein relation correspond to smoothings of the crossing. A smoothing is associated to a marker, which specifies a pair of vertical angles at the crossing to be joined under the smoothing (see Figure 1).

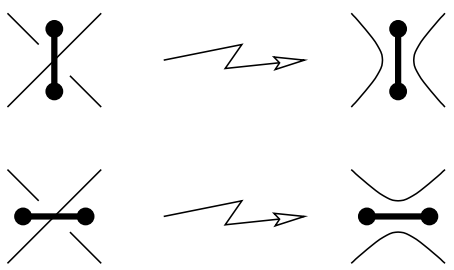

Fig. 1. Smoothing of a diagram according to markers

Applying the skein relation at all crossings gives rise to a presentation of $\langle D\rangle$ as a large sum in which the summands correspond to distributions of markers over all crossings. Such a distribution of markers is called a state of the diagram. A state of a diagram defines a smoothing of the diagram: at each of its double points the marked angles are united in a connected area.

Denote the result of the smoothing by $D_{s}$. This is a union of disjoint circles embedded in the plane. Denote the number of the circles by $|s|$. 
The two terms on the right hand side of the Kauffman skein relation have different coefficients $\left(A\right.$ and $A^{-1}$ ). The corresponding markers are also distinct: the one corresponding to the term with coefficient $A$ connects the verticle angles such that rotation of the upper string towards the lower one through these angles is counter-clockwise, while for the other the rotation is clockwise. The markers of the former kind are called positive or A-markers, those of the latter kind are negative or $B$-markers. Khovanov [7] calls the smoothing corresponding to an $A$-marker a 0 -resolution, and the smoothing corresponding to a $B$-marker a 1-resolution. Bar-Natan [2] calls them 0smoothing and 1-smoothing, respectively. See Figure 2.

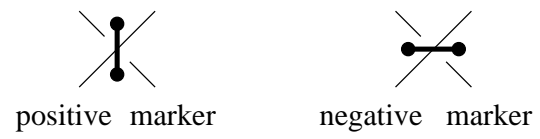

Fig. 2. Markers

For a state $s$ of a diagram $D$ denote by $\sigma(s)$ the difference between the numbers of positive and negative markers. The contribution of a state $s$ to the Kauffman bracket is $A^{\sigma(s)}\left(-A^{2}-A^{-2}\right)^{|s|-1}$ and

$$
\langle D\rangle=\sum_{\text {states } s \text { of } D} A^{\sigma(s)}\left(-A^{2}-A^{-2}\right)^{|s|-1} .
$$

Together with (1), this gives

$$
V_{D}\left(A^{-4}\right)=(-A)^{-3 w(D)} \sum_{\text {states } s \text { of } D} A^{\sigma(s)}\left(-A^{2}-A^{-2}\right)^{|s|-1} .
$$

2.4. Jones polynomial and Kauffman bracket augmented. The normalization adopted in the classical definitions implies that for the empty link $\varnothing$ the Jones polynomial and the Kauffman bracket are not Laurent polynomials:

$$
V_{\varnothing}=\frac{1}{-t^{1 / 2}-t^{-1 / 2}} \quad \text { and } \quad\langle\varnothing\rangle=\frac{1}{-A^{2}-A^{-2}} .
$$

To turn them into Laurent polynomials, as well as for many other reasons, the normalization property is modified by requiring that the Jones polynomial and Kauffman bracket of the empty link are 1. This gives rise to a new version of the Jones polynomial which is equal to the original one multiplied by $-t^{1 / 2}-t^{-1 / 2}$, and a new version of the Kauffman bracket which is the original one multiplied by $-A^{2}-A^{-2}$. In particular, the new version of the Jones polynomial of the unknot equals $-t^{1 / 2}-t^{-1 / 2}$ and the new version of the Kauffman bracket of the unknot with 0-framing equals $-A^{2}-A^{-2}$.

We will call these versions augmented and denote them by $\widetilde{V}_{L}$ and $\widetilde{\langle L\rangle}$, respectively. 
The state sum representations (2) and (3) imply

$$
\begin{gathered}
\widetilde{\langle D\rangle}=\sum_{\text {states } s \text { of } D} A^{\sigma(s)}\left(-A^{2}-A^{-2}\right)^{|s|}, \\
\widetilde{V}_{D}\left(A^{-4}\right)=(-A)^{-3 w(D)} \sum_{\text {states } s \text { of } D} A^{\sigma(s)}\left(-A^{2}-A^{-2}\right)^{|s|} .
\end{gathered}
$$

2.5. Terminological remarks. In the literature one can find several normalizations of both the Jones polynomial and the Kauffman bracket. They differ by factors and change of variable. The terminology concerning the Jones polynomial and Kauffman bracket is a bit messy. I will consider here only issues related directly to the construction of Khovanov homology.

In the paper [2] by Bar-Natan the term Kauffman bracket is used for another characteristic of a link diagram, which fails to be invariant under the second Reidemeister move.

This bracket was borrowed from Khovanov's paper [7], where it was not named at all, but enjoyed the bracket notation.

Khovanov used the variable $q=-A^{-2}$. In this variable the Kauffman bracket is a Laurent polynomial in $q^{1 / 2}$ with coefficients in the Gaussian numbers $\mathbb{Z}[\sqrt{-1}]$. Probably, just to keep the Kauffman bracket a Laurent polynomial in $q$ with integer coefficients, Khovanov multiplied it by $(-q)^{c / 2}$, where $c$ is the number of crossings in the diagram. This compromised its invariance under the second Reidemeister move, but the invariance was not needed.

In Khovanov's paper [7], the term "Kauffman bracket" was used for a rescaled version of the Jones polynomial $f[L]$ introduced by Kauffman in [6] for a short while, just to prove Theorem 2.8 there, which states that $f[K]$ evaluated at $t^{-1 / 4}$ is the Jones polynomial $V_{K}(t)$ of $K$.

Kauffman in [6] surely did not use the words "Kauffman bracket", but he used the term "bracket polynomial" for a polynomial characteristic of a link diagram and denoted it with brackets. The name of Kauffman bracket was then commonly accepted for this polynomial. This is what we call Kauffman bracket.

There is nothing wrong in modifying polynomial characteristics of link diagrams according to current needs, provided this does not lead to confusion. I suggest following the original terminology as closely as possible, up to notations of indeterminates in the polynomials.

3. Preliminary reflections on categorifications. We are not going to discuss the general idea of categorification. Instead, let us concentrate on classical non-trivial examples, which are most instructive for our purposes. 
3.1. Classical examples of categorification. A classical example of categorification is the invention of homology groups. Homology groups categorify the Euler characteristic. The process of categorification took quite a long time and efforts of excellent mathematicians such as Betti, Riemann, Poincaré, Vietoris and Emmi Noether. One can hope to find an inspiration in this example.

The homology groups $H_{n}(X)$ of a space $X$ categorify the Euler characteristic $\chi(X)$ in the sense that

$$
\chi(X)=\sum_{n=0}^{\operatorname{dim} X}(-1)^{n} \operatorname{rk} H_{n}(X) .
$$

Formula (6) means that the Euler characteristic characterizes numerically the size of the homology groups. So, if we begin with the Euler characteristic, the homology groups provide an answer to the question: "What does the Euler characteristic count?", or, more accurately: "What is an algebraic invariant of a topological space such that the size of this invariant is characterized by the Euler characteristic?"

The Euler characteristic of, say, a polyhedron $X$ can be defined by

$$
\chi(X)=\sum_{\substack{\text { simplices } s \text { of } \\ \text { a triangulation of } X}}(-1)^{\operatorname{dim}(s)} .
$$

This is a version of the well known formula

$$
\chi(X)=\sum_{n=0}^{\operatorname{dim} X}(-1)^{n} c_{n}(X),
$$

where $c_{n}(X)$ is the number of $n$-dimensional simplices in a triangulation of $X$. So, the Euler characteristic counts simplices taking into account the parity of their dimensions. However, the numbers $c_{n}(X)$ depend on triangulation, while $\chi(X)$ does not.

The homology groups $H_{n}(X)$ do not depend on triangulation. To construct them, one can first construct the chain groups $C_{n}(X)$. Recall that $C_{n}(X)$ is a free abelian group generated by $n$-dimensional simplices of some fixed triangulation of $X$. This simple formal action seems to be a step in the right direction. Indeed,

$$
\chi(X)=\sum_{n=0}^{\operatorname{dim} X}(-1)^{n} \operatorname{rk} C_{n}(X),
$$

because $c_{n}(X)=\operatorname{rk} C_{n}(X)$. However, the groups $C_{n}(X)$ depend not only on $X$, but on the choice of the triangulation.

A crucial step is to pass from chains to homology classes. One needs to find boundary operators $\partial_{n}: C_{n}(X) \rightarrow C_{n-1}(X)$ such that the compositions 
$\partial_{n-1} \circ \partial_{n}$ would be trivial and the homology groups $H_{n}(X)=\operatorname{Ker} \partial_{n} / \operatorname{Im} \partial_{n+1}$ would not depend on the triangulation. As soon as this is achieved, we are done, since for any complex $C=\left\{C_{N} \rightarrow C_{N-1} \rightarrow \cdots \rightarrow C_{0}\right\}$ of abelian groups,

$$
\sum_{n=0}^{N}(-1)^{n} \operatorname{rk} H_{n}(C)=\sum_{n=0}^{N}(-1)^{n} \operatorname{rk} C_{n} .
$$

The advantages of homology groups over the Euler characteristic are well known. Homology groups certainly contain much more information about the space than the Euler characteristic. But the functorial nature of homology is even more important. Continuous maps between spaces induce homomorphisms between their homology groups. Without homology groups very little can be said about continuous maps in terms of just Euler characteristics.

3.2. Non-uniqueness of categorification. A categorification of the Euler characteristic is not unique. For example, one can consider homology groups with coefficients in a field $F$. Ranks of groups should then be replaced by dimensions over $F$, so formula (6) turns into

$$
\chi(X)=\sum_{n=0}^{\operatorname{dim} X}(-1)^{n} \operatorname{dim}_{F} H_{n}(X ; F) .
$$

Even more categorifications of $\chi(X)$ can be obtained by cheap tricks. For example, one can change the indices (dimensions) of the homology groups preserving only their parity. Or one can choose any finite sequence of finitely generated abelian groups $A_{k}$ with $\sum_{k}(-1)^{k} \mathrm{rk} A_{k}=1$ and put $H_{n}^{A}(X)=$ $\bigoplus_{k}\left(A_{k} \otimes H_{n-k}(X)\right)$. Obviously, $\chi(X)=\sum_{n}(-1)^{n} \operatorname{rk} H_{n}^{A}(X)$.

3.3. How should a categorification of a polynomial link invariant look like?. We are going to categorify the augmented Jones polynomial and the augmented Kauffman bracket. Both are Laurent polynomials in one variable over $\mathbb{Z}$. A Laurent polynomial in one variable is nothing but a sequence of its coefficients.

One may expect that under categorification, each coefficient, being a numerical invariant of a link, gives rise to a sequence of abelian groups. Therefore a categorification of the whole polynomial should look like a collection of abelian groups indexed by pairs of numbers. Say, a categorification of the augmented Jones polynomial is expected to be a collection of abelian groups $H^{i, j}(L)$ such that $\widetilde{V}_{L}(t)=\sum_{i, j}(-1)^{i} t^{j} \mathrm{rk} H^{i, j}(L)$.

Instead of a polyhedron, we now have to deal with a link. Triangulation that served a combinatorial presentation of a polyhedron, is replaced by a link diagram. 
The Kauffman state sum representation (5) of the Jones polynomial looks similar to the formula (7) for the Euler characteristic in terms of numbers of simplices. To make it a true counterpart of (7), each homogeneous component of the right hand side of (5) should be represented as an alternating sum of ranks of groups for a sequence of abelian groups. Then the groups next to each other in this sequence should be related by homomorphisms, transforming the sequence into a chain complex whose homology groups do not change under Reidemeister moves.

We are going to realize this program.

\section{From Kauffman bracket to Khovanov chains}

4.1. Normalization problem: homology for the unknot. Since categorification is not unique, we should feel free to impose additional restrictions, a sort of normalization, on $H^{i, j}(L)$.

The normalization property of the augmented Jones polynomial states that for the unknot it equals $-t^{1 / 2}-t^{-1 / 2}$. Thus

$$
\sum_{i}(-1)^{i} \mathrm{rk} H^{i, j} \text { (unknot) }= \begin{cases}-1 & \text { if } j= \pm 1 / 2 \\ 0 & \text { otherwise. }\end{cases}
$$

There is no aesthetically obvious choice for $H^{i, j}$ (unknot) to satisfy this restriction. One of the simplest choices is $H^{i, j}=\mathbb{Z}$ if $i=1$ and $j= \pm 1 / 2$, and $H^{i, j}=0$ otherwise. However, there are other possibilities, which are, maybe, not worse, if not better than this one. Say, non-trivial groups may appear at $i=1, j=1 / 2$ and $i=-1, j=-1 / 2$.

4.2. Khovanov's change of variable. Maybe, this ambiguity was the point which forced Khovanov to change variable in the augmented Jones polynomial. He replaced $t$ with $q=-t^{1 / 2}$. Following Khovanov, let us denote by $K(L)$ the polynomial in $q$ obtained in this way. It is defined by the following properties:

(1) Normalization. $K($ unknot $)=q+q^{-1}$.

(2) Stabilization. $K(L \amalg$ unknot $)=\left(q+q^{-1}\right) K(L)$.

(3) Skein relation. $q^{-2} K\left(\aleph^{\top}\right)-q^{2} K\left(\chi^{1}\right)=\left(q^{-1}-q\right) K()()$.

Now the simplest possibility for the value of categorification of $K$ on the unknot is obvious:

$$
H^{i, j}= \begin{cases}\mathbb{Z} & \text { if } i=0 \text { and } j= \pm 1 \\ 0 & \text { otherwise }\end{cases}
$$

As we have chosen to categorify $K(L)$ instead of $\widetilde{V}_{L}$, we want to find, for each link $L$, a collection of abelian groups $\mathcal{H}^{i j}(L)$ such that $K(L)=$ $\sum_{i, j}(-1)^{i} q^{j} \mathrm{rk} \mathcal{H}^{i, j}(L)$. (We have switched to Khovanov's notation, because 
now we are really talking about the homology groups $\mathcal{H}^{i, j}$ he has constructed.)

4.3. Enhanced states. The Kauffman state sum (5) for the augmented Jones polynomial of a framed link $L$ presented by a link diagram $D$ with blackboard framing turns into

$$
K(L)(q)=\sum_{\text {states } s \text { of } D}(-1)^{(w(D)-\sigma(s)) / 2} q^{(3 w(D)-\sigma(s)) / 2}\left(q+q^{-1}\right)^{|s|} .
$$

The summands on the right hand side of (11) correspond to states of the diagram. Hence each summand has a geometric meaning. Unfortunately, each of them contributes to several monomials of $K(L)$, and therefore it should contribute to several homology groups. Therefore states cannot be considered as true counterparts of simplices from the categorification of the Euler characteristic considered in 3.1. We need to invent refinements of states which would contribute monomials.

The most obvious straightforward way to refine the states is to open brackets in $\left(q+q^{-1}\right)^{|s|}$ and associate the monomials to some geometric objects.

The smoothened diagram $D_{s}$ consists of $|s|$ connected components. So, one can associate each factor $q+q^{-1}$ of $\left(q+q^{-1}\right)^{|s|}$ with one of the components. A monomial of the sum obtained by opening brackets in the product corresponds to a choice of $q$ or $q^{-1}$ in each of the factors. This gives rise to the following definition.

By an enhanced state $S$ of a link diagram $D$ we shall mean a collection of markers constituting a usual Kauffman state $s$ of $D$ enhanced by an assignment of a plus or minus sign to each of the circles of $D_{s}$. (Recall that $D_{s}$ is obtained by smoothing $D$ according to all markers of $s$.)

For an enhanced state $S$ of an oriented link diagram $D$ denote by $\tau(S)$ the difference between the numbers of pluses and minuses assigned to the circles of $D_{s}$. Observe that for any state $s$ of $D$,

$$
q^{(3 w(D)-\sigma(s)) / 2}\left(q+q^{-1}\right)^{|s|}=\sum_{\substack{\text { enhanced states } S \\ \text { enhancing } s}} q^{(3 w(D)-\sigma(s)+2 \tau(S)) / 2}
$$

Put $\left({ }^{1}\right)$

$$
j(S)=\frac{3 w(D)-\sigma(s)+2 \tau(S)}{2} .
$$

Observe that both $\sigma(s)$ and $w(D)$ are congruent modulo 2 to the number of crossing points. Therefore $j(S)$ is an integer. Substituting (12) to (11), we

$\left({ }^{1}\right)$ In [18] the formula for $j(S)$ was slightly different: $j(S)=\frac{1}{2}(3 w(D)-\sigma(s)-2 \tau(S))$. This was caused by the opposite value of the sign of the circles in $D_{s}$. In [18] the change of Kauffman's variable $A$ to Khovanov's variable $q=-A^{-2}$ was made after the definition of $\tau(S)$. 
get

$$
K(L)(q)=\sum_{\text {enhanced states } S \text { of } D}(-1)^{(w(D)-\sigma(s)) / 2} q^{j(S)} .
$$

4.4. Khovanov chain groups. Denote a free abelian group generated by the enhanced states of a link diagram $D$ by $\mathcal{C}(D)$. Denote by $\mathcal{C}^{j}(D)$ the subgroup of $\mathcal{C}(D)$ generated by the enhanced states $S$ of $D$ with $j(S)=j$. Thus $\mathcal{C}(D)$ is a $\mathbb{Z}$-graded free abelian group:

$$
\mathcal{C}(D)=\bigoplus_{j \in \mathbb{Z}} \mathcal{C}^{j}(D)
$$

For an enhanced state $S$ belonging to a state $s$ of a link diagram $D$, put

$$
i(S)=\frac{w(D)-\sigma(s)}{2} .
$$

Denote by $\mathcal{C}^{i, j}(D)$ the subgroup of $\mathcal{C}^{j}(D)$ generated by the enhanced states $S$ with $i(S)=i$. Notice that it follows from (13) that

$$
K(L)(q)=\sum_{j=-\infty}^{\infty} q^{j} \sum_{i=-\infty}^{\infty}(-1)^{i} \mathrm{rk}^{i, j}(D) .
$$

4.5. Linguistic differences to Bar-Natan's formalism. Enhancements of states replace other notions, which appeared to be central in Khovanov's construction [7] and its presentation by Bar-Natan [2]. We consider a smaller set of notions than in Bar-Natan's paper [2].

Briefly, graded groups are used extensively at intermediate stages of the construction in [7] and [2].

The states of a link diagram $D$ with $n$ crossings correspond to the vertices of the $n$-dimensional cube. To define this correspondence, one has to fix a (total) order of crossings and associate to a state $s$ an $n$-vector $\left(\varepsilon_{1}, \ldots, \varepsilon_{n}\right)$, where $\varepsilon_{i}=0$ if the marker of $s$ at the $i$ th crossing is positive, and $\varepsilon_{i}=1$ otherwise.

With every vertex $\alpha \in\{0,1\}^{n}$ of the $n$-dimensional cube (i.e., with every state $s$ of $D$ ) Bar-Natan associated $\left({ }^{2}\right)$ a graded free abelian group $V_{\alpha}$. In our terms, $V_{\alpha}$ is a free abelian group generated by the enhancements of the state $s$ corresponding to $\alpha \in\{0,1\}^{n}$. The grading in $V_{\alpha}$ is defined by the numbers $i(S)$ introduced above, in 4.4, for each enhancement $S$ of $s$. The homogeneous component of $V_{\alpha}$ of degree $i$ is the subgroup generated by the enhanced states $S$ with $i(S)=i$.

$\left(^{2}\right)$ Bar-Natan wrote about graded vector spaces, but mentioned in a footnote: "everything that we do works just fine (with some linguistic differences) over $\mathbb{Z}$ ". We switch tacitly back to abelian groups, i.e., modules over $\mathbb{Z}$, but in the next section we will switch for a short while to vector spaces over $\mathbb{Z}_{2}$. 
The original construction of $V_{\alpha}$ runs through several steps with more algebraic details, which are useful in what follows.

First, Bar-Natan introduced a free abelian group $V$ of rank 2 with generators $v_{ \pm}$. They are of degrees \pm 1 respectively. This turns $V$ into a graded group: $V=V_{+1} \oplus V_{-1}$, where $V_{ \pm 1}$ is the subgroup of $V$ generated by $v_{ \pm}$.

By the graded rank of a graded abelian group $W=\bigoplus_{m} W_{m}$ with homogeneous components $W_{m}$ we mean the power series qrk $W=\sum_{m} q^{m} \mathrm{rk} W_{m}$. For example, qrk $V=q+q^{-1}$.

From our perspective, $v_{+}$and $v_{-}$are the two enhancements of a state $s$ with $|s|=1$. In general, to construct $V_{\alpha}$ Bar-Natan [2] (following Khovanov [7]) takes the $|s|$ th tensor power of $V$ and shifts the grading appropriately.

The $|s|$ th tensor power $V^{\otimes|s|}$ is generated by $v_{\delta_{1}} \otimes \cdots \otimes v_{\delta_{|s|}}$, where $\delta_{i}= \pm$. Obviously, qrk $V^{\otimes|s|}=\left(q+q^{-1}\right)^{|s|}$. The $q$-rank of $V_{\alpha}$ has to be the contribution of the state $s$ to $K(L)$, that is, qrk $V_{\alpha}=q^{(3 w(D)-\sigma(s)) / 2}(q+$ $\left.q^{-1}\right)^{|s|}$. The group $V^{\otimes|s|}$ is turned into $V_{\alpha}$ just by shifting the grading by $(3 w(D)-\sigma(s)) / 2$.

Let us introduce a notation for the shifting. Let $\cdot\{l\}$ denote the degree shift operation: for $W=\bigoplus_{m} W_{m}$ set $W\{l\}_{m}=W_{m-l}$ and $W\{l\}=$ $\bigoplus_{m} W\{l\}_{m}$, so that qrk $W\{l\}=q^{l}$ qrk $W$. With this notation,

$$
V_{\alpha}=\left(V^{\otimes|s|}\right)\{(3 w(D)-\sigma(s)) / 2\} .
$$

Let us associate factors of $V^{\otimes|s|}=V \otimes \cdots \otimes V$ with components of $D_{s}$. Each of the standard generators of $V^{\otimes|s|}$ is associated with an enhancement of $s$ in the obvious way: a generator $v_{\delta_{1}} \otimes \cdots \otimes v_{\delta_{|s|}}$ corresponds to the enhancement of $s$ in which the $i$ th component of $D_{s}$ is equipped with sign $\delta_{i}$.

5. From chains to homology. Let $D$ be a diagram of an oriented link $L$. In [7] Khovanov defined a differential of bidegree $(1,0)$ in $\mathcal{C}^{i, j}(D)$ and proved that the corresponding homology groups $\mathcal{H}^{i, j}(D)$ depend, up to isomorphism, only on $L$.

Khovanov's description of the differential is made somewhat complicated by several auxiliary algebraic constructions. We consider here a simplified version, first over $\mathbb{Z}_{2}$.

An impatient (or lazy) topologist tends to consider homology with coefficients in $\mathbb{Z}_{2}$ instead of the full-fledged homology with integer coefficients. This is a decent way to make a rewarding part of job. Its result is an indispensible step towards constructing the homology with integer coefficients, but all difficulties related to signs are delayed.

In the case of Khovanov homology restricting ourselves to considerations modulo 2 we do not even miss our goal to categorify $K(L)$. Indeed, the homology groups of $\mathcal{C}\left(D ; \mathbb{Z}_{2}\right)=\mathcal{C}(D) \otimes \mathbb{Z}_{2}$ denoted by $\mathcal{H}^{i, j}\left(D ; \mathbb{Z}_{2}\right)$ already 
categorify $K(L)$ in the sense that

$$
K(L)=\sum_{i, j}(-1)^{i} q^{j} \operatorname{dim}_{\mathbb{Z}_{2}} \mathcal{H}^{i, j}\left(D ; \mathbb{Z}_{2}\right) .
$$

5.1. Incidence numbers modulo 2. To define the differential $d_{2}$ of $\mathcal{C}\left(D ; \mathbb{Z}_{2}\right)$, we just describe the matrix elements for $d_{2}$. In the context of chain complexes the matrix elements are traditionally called incidence numbers.

The group $\mathcal{C}^{i, j}\left(D ; \mathbb{Z}_{2}\right)$ is generated by $S \otimes 1$, where $S$ runs over enhanced states of $D$ with $i(S)=i$ and $j(S)=j$. We will denote $S \otimes 1$ by $S$, as this causes no confusion and simplifies notation.

For enhanced states $S$ and $T$, denote their incidence number modulo 2 by $(S: T)_{2}$. Then for an enhanced state $S$,

$$
d_{2}(S)=\sum_{\text {enhanced states } T}(S: T)_{2} T .
$$

The incidence number $(S: T)_{2}$ is a function of the pair of enhanced states $S$ and $T$, which are generators of $\mathcal{C}^{i, j}\left(D ; \mathbb{Z}_{2}\right)$ and $\mathcal{C}^{i+1, j}\left(D ; \mathbb{Z}_{2}\right)$, respectively.

5.2. Restrictions on pairs of incident enhanced states. Enhanced states with a non-zero incidence number are said to be incident to each other. Pairs of incident states satisfy natural restrictions. Surprisingly, these restrictions give an exact description of the set of incident states: each pair of enhanced states which is not eliminated by the restrictions consists of incident states.

5.2.A. If $S$ and $T$ are enhanced states with $(S: T)_{2} \neq 0$, then $j(T)=$ $j(S)$ and $i(T)=i(S)+1$.

Proof. This restriction emerges from our desire to have a differential of bidegree $(1,0)$. Thus the differential preserves $j$ and increases $i$ by one.

5.2.B. Corollary. If $S$ and $T$ are enhanced states with $(S: T)_{2} \neq 0$, then $\sigma(T)=\sigma(S)-2$.

Proof. Recall that $i(S)=(w(D)-\sigma(S)) / 2$. Therefore $i(T)=i(S)+1$ implies $\sigma(T)=\sigma(S)-2$. In other words, the number of negative markers of $T$ is one greater than the number of negative markers of $S$.

It is natural to enforce this numerical restriction in the following way:

5.2.C. Assumption on InCIDEnt States. The incidence number $(S: T)_{2}$ is zero unless the markers of $S$ and $T$ differ at only one crossing point of $D$, and at this crossing the marker of $S$ is positive, and that of $T$ is negative.

The crossing where the markers of $S$ and $T$ satisfying 5.2.C differ is called the changing crossing of $S$ and $T$ and denoted by $x(S, T)$. 


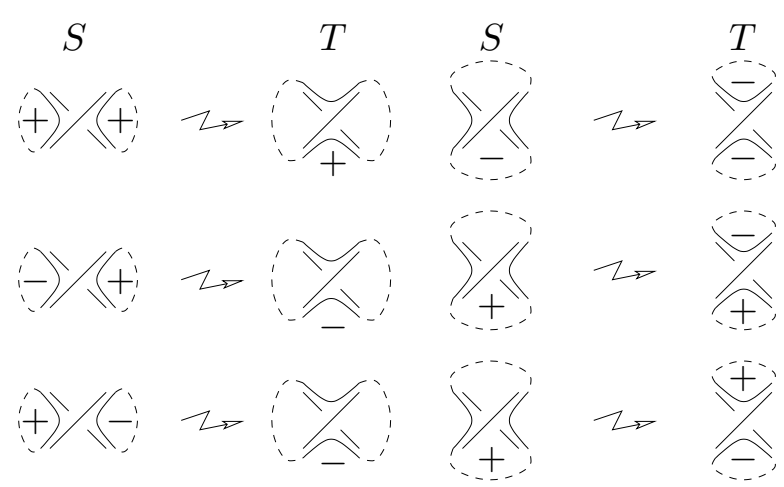

Fig. 3. Incident enhanced states. The dotted arcs show how fragments of $D_{S}$ or $D_{T}$ are connected in the whole $D_{S}$ or $D_{T}$

Since the markers differ at exactly one crossing, $D_{T}$ is obtained from $D_{S}$ by a single Morse modification of index 1 . Since the Morse modification is embedded in the plane, it respects the orientations of $D_{S}$ and $D_{T}$ induced from the domains bounded by $D_{S}$ and $D_{T}$ in the plane. Hence $|S|-|T|= \pm 1$. In other words, $D_{T}$ is obtained from $D_{S}$ either by joining two circles or by splitting a circle of $D_{S}$ into two circles.

Here is the next natural restriction on incident enhanced states:

5.2.D. Assumption on incident enhancements. Enhanced states $S$ and $T$ are not incident unless the common components of $D_{S}$ and $D_{T}$ have the same signs in $S$ and $T$.

5.2.E. Corollary $\left(^{3}\right)$. If $S$ and $T$ are enhanced states with $(S: T)_{2}$ $\neq 0$, then $\tau(T)=\tau(S)-1$.

Proof. Indeed, $j(T)=j(S)$ by 5.2.A, and $\sigma(T)=\sigma(S)-2$ by 5.2.B. Hence

$$
\begin{aligned}
j(T) & =\frac{3 w(D)-\sigma(T)+2 \tau(T)}{2} \\
& =j(S)=\frac{3 w(D)-\sigma(S)+2 \tau(S)}{2}=\frac{3 w(D)-\sigma(S)-2+2 \tau(S)}{2} .
\end{aligned}
$$

Now we can list all situations satisfying these restrictions (see Figure 3):

5.2.F. Corollary. Let $S$ and $T$ be enhanced states with $(S: T)_{2} \neq 0$.

(1) If $|T|=|S|-1$ and both joining circles of $D_{S}$ are positive then the resulting circle of $T$ should be positive.

(2) If $|T|=|S|-1$ and the joining circles of $D_{S}$ have different signs then the resulting circle of $T$ should be negative.

$\left({ }^{3}\right)$ This statement differs from the corresponding statement in [18] due to the difference in the sign of $\tau$. 
(3) If $|T|=|S|+1$ and the splitting circle of $D_{S}$ is negative then both of the circles of $D_{T}$ obtained from it should be negative.

(4) If $|T|=|S|+1$ and the splitting circle of $D_{S}$ is positive then the circles of $D_{T}$ obtained from it should be of different signs.

5.3. Differential modulo 2. Define $d_{2}: \mathcal{C}^{i, j}\left(D ; \mathbb{Z}_{2}\right) \rightarrow \mathcal{C}^{i+1, j}\left(D ; \mathbb{Z}_{2}\right)$ by assuming $(S: T)_{2}=1$ in each of the cases listed in 5.2.F and shown in Figure 3.

Another, more algebraic, description can be found in [7] and [2]. The graded space $\bigoplus_{i, j} \mathcal{C}^{i, j}\left(D ; \mathbb{Z}_{2}\right)$ can be identified with

$$
\bigoplus_{\text {states } s \text { of } D}\left(V \otimes \mathbb{Z}_{2}\right)^{\otimes|s|}\left\{\frac{3 w(D)-\sigma(s)}{2}\right\}
$$

(see Section 4.4). The factors in each summand are associated with connected components of $D_{s}$. The signs on the components are associated with the generators of the corresponding factor. Figure 3 turns into a description of two maps. The left hand side of Figure 3 describes a multiplication

$$
m: V \otimes V \rightarrow V, \quad m: \begin{cases}v_{+} \otimes v_{-} \mapsto v_{-}, & v_{+} \otimes v_{+} \mapsto v_{+}, \\ v_{-} \otimes v_{+} \mapsto v_{-}, & v_{-} \otimes v_{-} \mapsto 0,\end{cases}
$$

and the right hand side a comultiplication

$$
\Delta: V \rightarrow V \otimes V, \quad \Delta:\left\{\begin{array}{l}
v_{+} \mapsto v_{+} \otimes v_{-}+v_{-} \otimes v_{+}, \\
v_{-} \mapsto v_{-} \otimes v_{-} .
\end{array}\right.
$$

Of course, these are the restrictions described in the preceding section that shape $m$ and $\Delta$.

5.3.A. Theorem. $d_{2}$ is a differential (i.e., $d_{2}^{2}=0$ ).

Proof. This can be proven as certain identities relating the multiplication $m$ and comultiplication $\Delta$ using the algebraic reformulation above (see [7]). These identities are: commutativity and associativity of $m$, cocommutativity and coassociativity of $\Delta$, and the identity $\Delta \circ m=(m \otimes \mathrm{id}) \circ(\mathrm{id} \otimes \Delta)$. However, the proof would involve checking numerous identities which were left as an exercise to the reader both in [7] and [2]. The algebraic reformulation helps only in naming the identities. A direct check involving only incidence numbers takes two pages, and we present it.

Since $d_{2}^{2}(S)=\sum_{T, U}(S: T)_{2}(T: U)_{2} U$, it is sufficient to prove that $\sum_{T}(S: T)_{2}(T: U)_{2}=0$ for each enhanced state $U$. A state $U$ for which this sum is not empty differs from $S$ by two markers. We can localize the problem by smoothing according to the common markers of $S$ and $T$, and forgetting those components of the result which do not pass through the marked crossings. At most three components of $D_{S}$ are left. 
Up to homeomorphism, only five possible pictures can appear:

(1) $D_{S}$ consists of three components and $D_{U}$ is connected, so the components of $D_{S}$ are united by two Morse modifications:

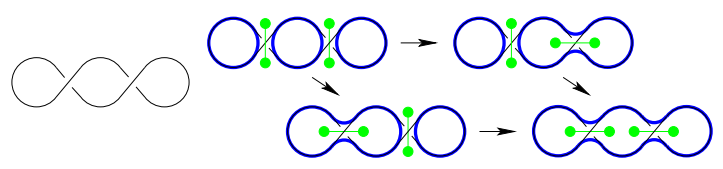

(2) $D_{S}$ is connected and $D_{U}$ consists of three connected components, so $D_{S}$ splits by two consecutive Morse modifications:

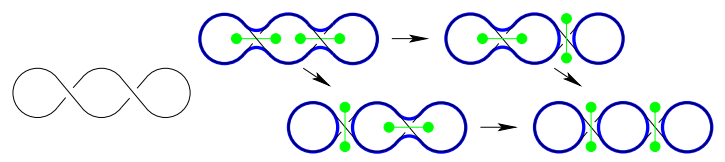

(3) Both $D_{S}$ and $D_{U}$ are connected, so $D_{S}$ splits by a Morse modification into two components, which are united by another Morse modification:
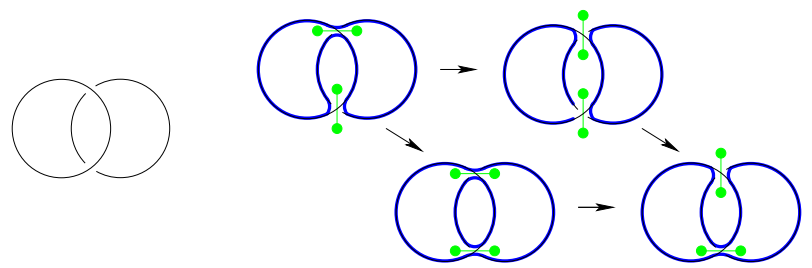

(4) Both $D_{S}$ and $D_{U}$ consist of two components; the first Morse modification unites the components of $D_{S}$, and the second splits the result:

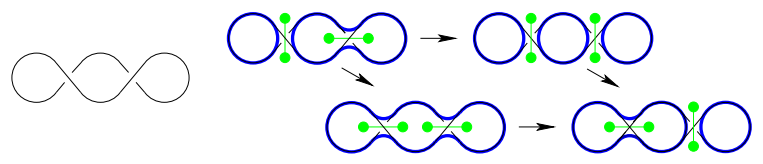

(5) Both $D_{S}$ and $D_{U}$ consist of two connected components; the first Morse modification splits one of the components of $D_{S}$ and then the second unites the other original component with one of the new components:
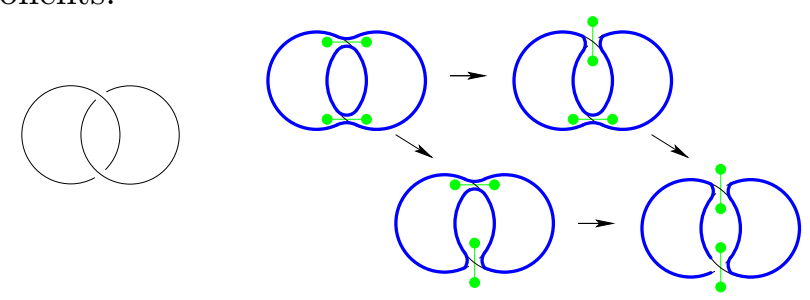

In each of these cases the order of the Morse modifications can be reversed. 
Then one can take into account enhancements, that is, the signs of the components obtained after the first Morse modification. One has to check that for any $S$ and $T$ the total number of ways to get $T$ from $S$ is even. All the possible distributions of signs on the pictures above such that enhancement states connected with an arrow are adjacent are shown below:
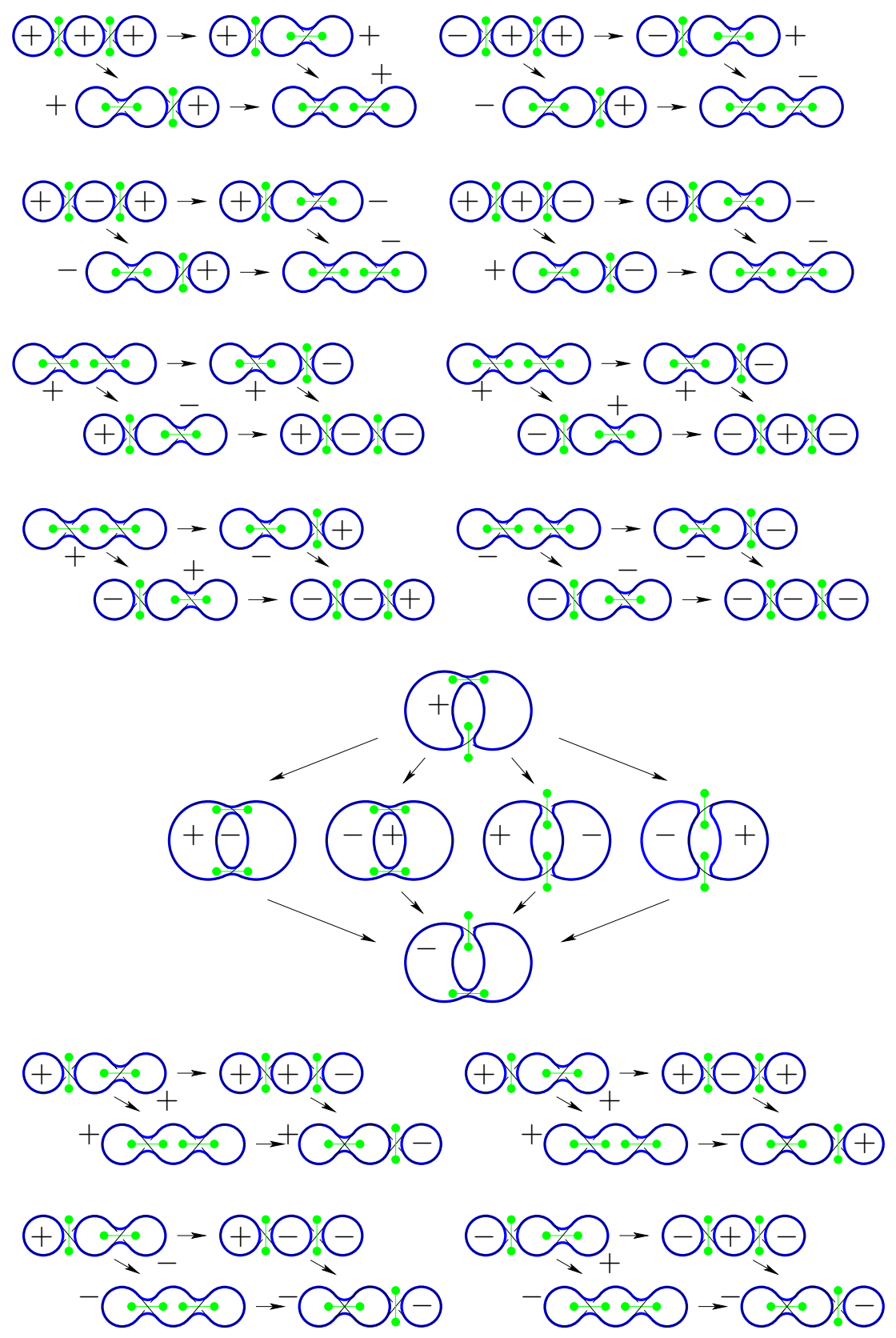

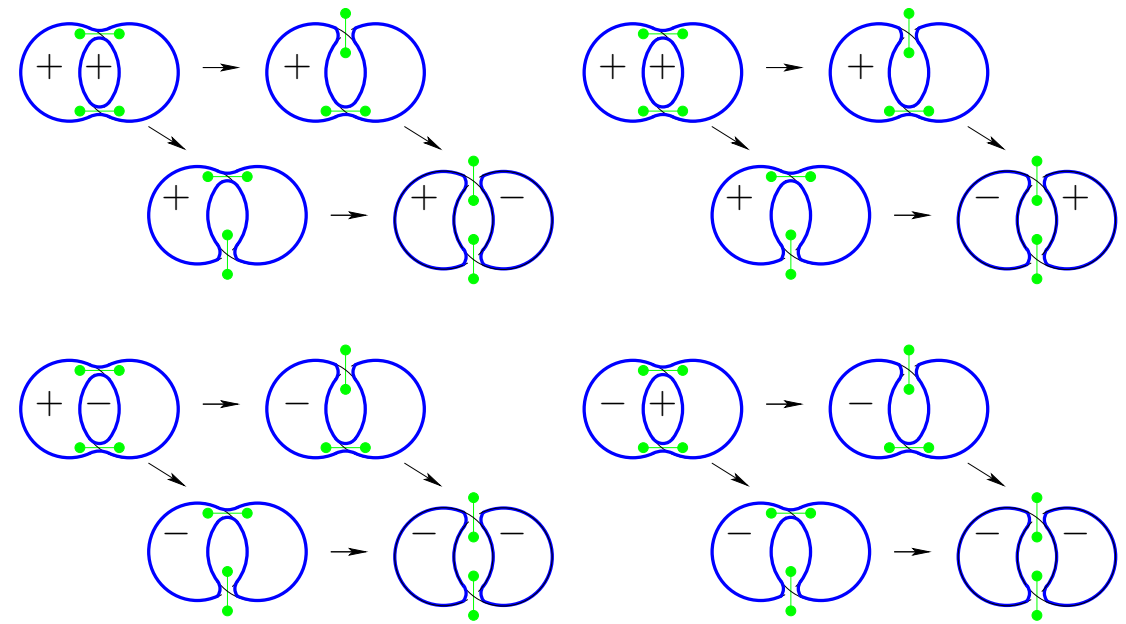

We see that if an enhanced state $S$ can be connected to an enhanced state $T$ by a chain of two adjacencies, there are an even number of such chains.

5.4. Upgrading to integer coefficients. To pass to integer coefficients, one can use the following three special properties of the adjacency of enhanced states.

5.4.A. For any pair of enhanced states $S$ and $T$ with $(S: T)_{2}=1$, there is exactly one crossing at which the markers of $S$ and $T$ differ, and at this crossing the marker of $S$ is positive.

Recall that for enhanced states $S, T$ with $(S: T)_{2}=1$, the crossing at which the markers of $S$ and $T$ differ is called the changing crossing and denoted by $x(S, T)$.

5.4.B. If $(S: T)_{2}=(T: U)_{2}=1$ then $x(S, T) \neq x(T, U)$.

Indeed, in $T$ the marker at $x(S, T)$ is negative, while the marker at $x(T, U)$ is positive.

5.4.C. For each pair of enhanced states $S, U$ such that there exists an enhanced state $T$ with $(S: T)_{2}=(T: U)_{2}=1$, on the set of such $T$ the changing crossing $x(S, T)$ takes two different values for an equal number of times.

These three properties of adjacency of enhanced states are analogous to the properties of adjacency for faces of a simplex. To use them, there are two standard ways, which are equivalent to each other.

First, one can order the crossings and define $(S: T)$ as $(-1)^{n}$, where $n$ is the number of negative markers in $S$ at crossings with numbers greater 
than $x(S, T)$. An obvious disadvantage of this approach is that it requires a proof of independence of homology from the ordering of the crossings.

Another approach is to define orientation of enhanced states and then define the incidence numbers for oriented enhanced states. By an orientation of an enhanced state we will mean a (linear) ordering of all negative markers considered up to even permutation. Orientations which differ by odd permutations are considered opposite. Orientations of enhanced states can be thought of as yet another enhancement of states, which allows one to define appropriate signs of adjacency indices. However, this enhancement does not increase the number of generators for the chain groups, since the same enhanced states with opposite orientations as chains differ by multiplication by -1 .

For enhanced states $S$ and $T$ with $(S: T)_{2}=1$ oriented by linear orderings of their negative markers such that the orderings coincide on the common markers followed by the changing crossing in the ordering for $T$, define $(S: T)=1$.

One can easily check that if the orientations of all enhanced states are defined by the orderings of negative markers induced by a linear order of all crossings then this definition of $(S: T)$ gives the same value as the definition of $(S: T)$ above.

5.4.D. Theorem. The homomorphism $d: \mathcal{C}^{i, j}(D) \rightarrow C^{i+1, j}(D)$ defined by $d(S)=\sum_{T}(S: T) T$ satisfies $d^{2}=0$.

Proof. Clearly, $d^{2}(S)=\sum_{U, T}(S: T)(T: U) U$; this is identically zero by 5.4.A-5.4.C and the definition of $(S: T)$.

5.5. Invariance under Reidemeister moves. Of course, the most fundamental property of the Khovanov homology groups is their invariance under Reidemeister moves. An explicit construction of chain maps inducing the corresponding isomorphisms can be found in paper [4] by Magnus Jacobsson. The same paper also contains an explicit description of the chain maps induced by a link cobordism.

One may wish a little bit more detailed information. Namely, the chain maps induced by a Reidemeister move are homotopy equivalences. Thus, there exist chain homotopy maps relating compositions of these maps with the identity maps. Below this is done for the case of first Reidemeister moves.

We have to distinguish two kinds of first Reidemeister moves: left-twisted $\supset=\rho$ and right-twisted $\supset$ - $>$.

5.6. Left-twisted first Reidemeister move. The Khovanov complex of the diagram obtained by a left-twisted first Reidemeister move splits as a direct sum of two subcomplexes, 


$$
\begin{aligned}
& \mathcal{C}(\bigcirc)=
\end{aligned}
$$

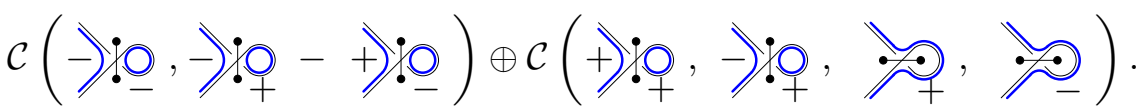

Here for the subcomplexes we use notation similar to the one used

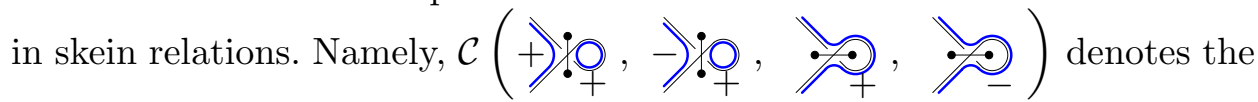
subcomplex generated (as a collection of groups) by all enhanced states which near the newborn crossing look as shown inside the parentheses.

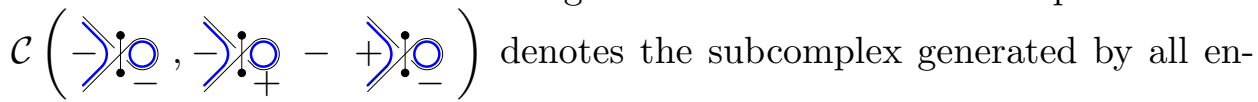
hanced states which near the newborn crossing look as $-\mathcal{H}_{-}$, and differences of two enhanced states which near the newborn crossing look as -10 and +.0 while outside of the neighborhood coincide with each other.

The first summand on the right hand side of (15) is isomorphic to a Khovanov complex of the diagram before the move. The isomorphism

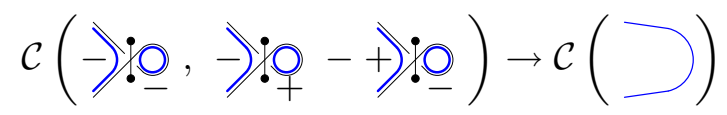

is defined by the formulas

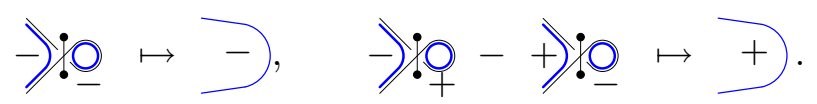

The second summand on the right hand side of (15) is contractible, as this is a cone over its subcomplex $\mathcal{C}(\stackrel{\bullet}{\rightleftharpoons})$. Thus, the sub-

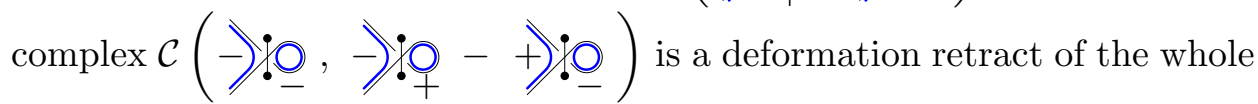

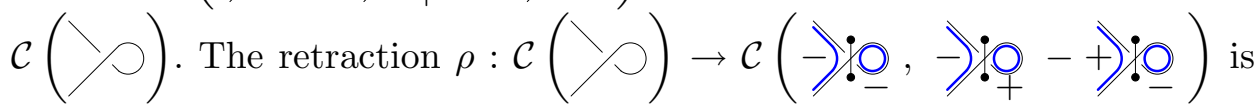
defined by the formulas

$$
\begin{aligned}
& -10 \mapsto-10
\end{aligned}
$$

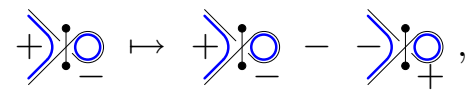

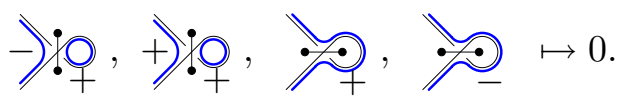

The homotopy connecting in $\circ \rho$ to the identity, that is, a map $h$ : $\mathcal{C}(\bigcirc) \rightarrow \mathcal{C}(\bigcirc)$ such that $d \circ h+h \circ d=\mathrm{id}-$ in $\circ \rho$, is defined 
by the formulas

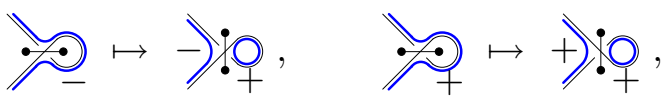

$$
\begin{aligned}
& +): 0,+) \div 0,->: 0,->\div 0 \mapsto 0 .
\end{aligned}
$$

Here we assume that the orientations of the enhanced states are defined by some order of crossings, in which the newborn crossing is the last one.

5.7. Right-twisted first Reidemeister move. The Khovanov complex of the diagram obtained by a right-twisted first Reidemeister move splits as a direct sum of two subcomplexes, too:

$$
\begin{aligned}
& \mathcal{C}(>)=
\end{aligned}
$$

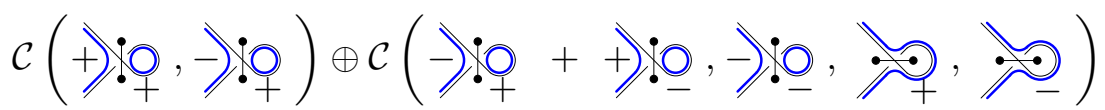

The first summand on the right hand side of (17) is isomorphic to the Khovanov complex of the diagram before the move. The isomorphism

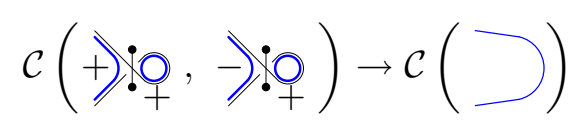

is defined by the formulas

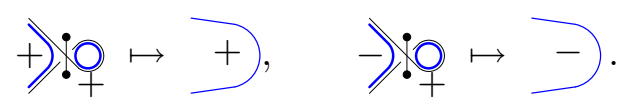

The second summand on the right hand side of (17) is contractible,

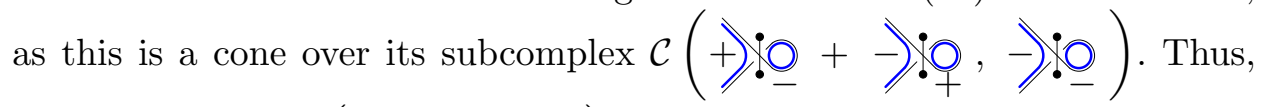

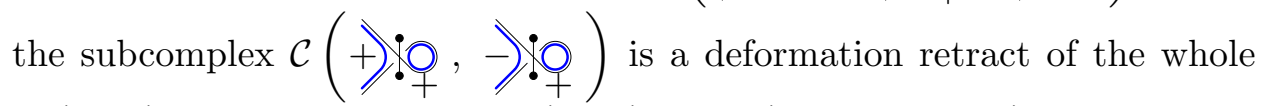

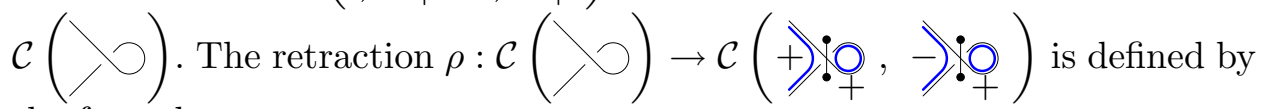
the formulas

$$
\begin{aligned}
& +): 0 \mapsto+): 0 \text {, } \\
& -): 0 \mapsto-7: 0, \\
& \text { +) }: 0 \mapsto->: 0,
\end{aligned}
$$

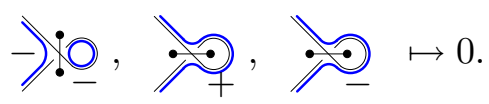


The homotopy connecting in $\circ \rho$ to the identity, that is, a map $h$ : $\mathcal{C}(\bigcirc) \rightarrow \mathcal{C}(\bigcirc)$ such that $d \circ h+h \circ d=\mathrm{id}-$ in $\circ \rho$, is defined by the formulas

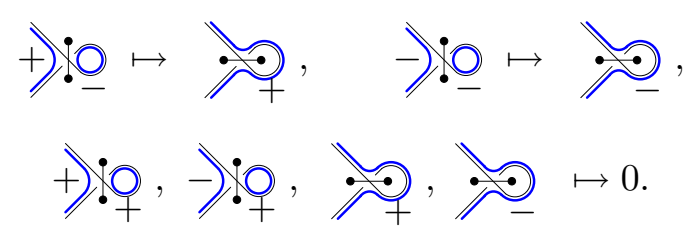

5.8. Enhanced states with polynomial coefficients. Khovanov constructed not only the groups $\mathcal{H}^{i, j}$, but also the graded modules $H^{i}$ over the ring $\mathbb{Z}[c]$ of polynomials with integer coefficients in variable $c$ of degree 2 . The grading is a representation of $H^{i}$ as a direct sum of abelian subgroups $H^{i, j}$ such that multiplication by $c$ in $H^{i}$ gives rise to a homomorphism $H^{i, j} \rightarrow H^{i, j+2}$.

To construct the homology groups $H^{i, j}$, we define the corresponding complex of graded $\mathbb{Z}[c]$-modules $C^{i}$. The module $C^{i}$ is the sum of its subgroups $C^{i, j}$. The group $C^{i, j}$ is generated by the formal products $c^{k} S$, where $k \geq 0$ and $S$ is an oriented enhanced state with $i(S)=i$ and $j(S)=j-2 k$.

The differential is defined in almost the same way as above. The states which were adjacent above are adjacent here, as are their products by the same power of $c$. Products of oriented enhanced states by different powers of $c$ are not adjacent, apart from the following situations: $c^{k+1} T$ is adjacent to $c^{k} S$ if the markers of $S$ and $T$ differ exactly at one point, where the marker of $S$ is positive and that of $T$ negative, the signs of $S$ and $T$ on the common circles of $D_{S}$ and $D_{T}$ are the same, $|T|=|S|+1$, the splitting circle of $D_{S}$ is negative, and the circles of $D_{T}$ obtained from it are positive; see Figure 4, where this situation is shown symbolically in the style of Figure 3.

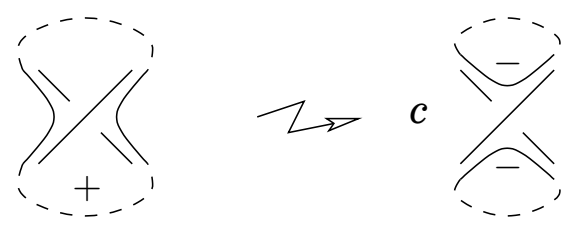

Fig. 4

Each group $C^{i, j}$ is finitely generated, but there are infinitely many nontrivial groups. The groups $H^{i, j}$ with fixed $i$ and sufficiently large $j$ are isomorphic to each other.

5.9. Lee's differentials. In [14] Eun Soo Lee defined a differential $\Phi$ of bidegree $(1,4)$ in the Khovanov complex. The corresponding adjacency of 
enhanced states is shown in Figure 5. She proved that $\Phi$ induces a homology operation in the Khovanov homology groups, and using this operation, established a conjecture on Khovanov homology of alternating knots formulated by Bar-Natan, Garoufalidis and Khovanov.
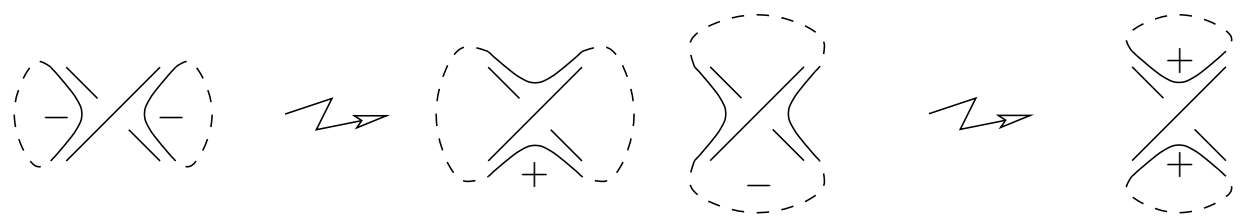

Fig. 5. Lee's differential $\Phi$

5.10. Reduced Khovanov homology. As observed by Khovanov [9], a categorification of the original, non-augmented, Jones polynomial can be obtained from the Khovanov complex by reduction. Again, Khovanov's description uses a bit more algebra than needed.

Here is a simplified version. I have learnt it from Alexander Shumakovitch (see [17]).

The reduced Khovanov chain complex is defined for a link diagram $D$ with a base point $d_{0}$ chosen on an $\operatorname{arc}$ of $D$.

To construct it, first consider a subcomplex $\widetilde{\mathcal{C}}\left(D, d_{0}\right)$ of $\mathcal{C}(D)$ generated by enhanced states $S$ such that the sign of the component of $D_{S}$ containing $d_{0}$ is - . The choice of - is not accidental. With - replaced by + it would not be a subcomplex.

The Euler characteristic of $\widetilde{\mathcal{C}}\left(D, d_{0}\right)$, that is, $\sum_{i, j}(-1)^{i} q^{j} \operatorname{rk} \widetilde{\mathcal{C}}^{i, j}\left(D, d_{0}\right)$, equals

$$
\frac{q^{-1}}{q+q^{-1}} K(D)=\frac{1}{q^{2}+1} K(D) .
$$

This is not $\frac{1}{q+q^{-1}} K(D)$, which is the polynomial obtained from the Jones polynomial $V_{D}(t)$ by the substitution $q=-t^{1 / 2}$. To fix this mismatch, we shift $\widetilde{\mathcal{C}}\left(D, d_{0}\right)$ appropriately: let $\mathcal{C}\left(D, d_{0}\right)$ be $\widetilde{\mathcal{C}}\left(D, d_{0}\right)\{1\}$. Denote the homology groups of $\mathcal{C}\left(D, d_{0}\right)$ by $\mathcal{H}^{i, j}\left(D, d_{0}\right)$.

Up to isomorphism, the groups $\mathcal{H}^{i, j}\left(D, d_{0}\right)$ depend only on the isotopy type of the oriented link with the marked component containing $d_{0}$.

The groups $\mathcal{H}^{i, j}\left(D, d_{0}\right)$ really depend on the component of the link containing $d_{0}$. The simplest example for which different base components give rise to non-isomorphic reduced Khovanov homology groups is a disjoint sum of the unknot and a trefoil knot.

6. Khovanov homology of framed links. In my preprint [18], I suggested a shifting turning the Khovanov homology of an oriented diagram into a categorification of the Kauffman bracket. For a link diagram $D$ it 
gives a bigraded collection of homology groups $H_{I, J}(D)$ such that

$$
\langle D\rangle(A)=\sum_{I, J}(-1)^{I / 2} A^{J} \operatorname{rk} H_{I, J}(D) .
$$

The shifting depends on the writhe number, and hence on orientation, while the groups do not depend on orientation, but depend on framing.

My choice of the grading was not satisfactory, and many people suggested to change it. Below I make another choice.

6.1. Framed Khovanov homology. Recall that the Kauffman bracket of a link diagram $D$ is given by the following state sum formula:

$$
\langle D\rangle=\sum_{\substack{\text { states } \\ s \text { of } D}} A^{\sigma(s)}\left(-A^{2}-A^{-2}\right)^{|s|}=\sum_{\substack{\text { enhanced states } \\ S \text { of } D}}(-1)^{|S|} A^{\sigma(S)-2 \tau(S)} .
$$

Observe that $|S| \equiv \tau(S)(\bmod 2)$, and hence

$$
\langle D\rangle=\sum_{\substack{\text { enhanced states } \\ S \text { of } D}}(-1)^{\tau(S)} A^{\sigma(S)-2 \tau(S)} .
$$

For an enhanced state $S$ of a link diagram $D$, put

$$
p(S)=\tau(S), \quad q(S)=\sigma(S)-2 \tau(S) .
$$

If the link is oriented, and hence $w(D)$ makes sense, then

$$
p(S)=j(S)-w(D)-i(S), \quad q(S)=3 w(D)-2 j(S) .
$$

In terms of $p(S)$ and $q(S)$, the Kauffman bracket is expressed as follows:

$$
\langle D\rangle=\sum_{\substack{\text { enhanced states } \\ S \text { of } D}}(-1)^{p(S)} A^{q(S)} .
$$

Denote the free abelian group generated by the enhanced states $S$ of $D$ with $p(S)=p$ and $q(S)=q$ by $\mathcal{C}_{p, q}(D)$. If one orients the link, the Khovanov chain groups $\mathcal{C}^{i, j}(D)$ appear, and

$$
\mathcal{C}_{p, q}(D)=\mathcal{C}^{(w(D)-q-2 p) / 2,(3 w(D)-q) / 2}(D) .
$$

Under this identification, the differentials of the Khovanov complex turn into differentials

$$
d: \mathcal{C}_{p, q}(D) \rightarrow \mathcal{C}_{p-1, q}(D)
$$

(the construction of the differentials does not involve the orientation of the link, hence it does not matter which orientation is used). Denote the homology group of the complex obtained by $H_{p, q}(D)$.

6.2. Skein homology sequence. Recall that the Kauffman bracket satisfies the following Kauffman skein relation:

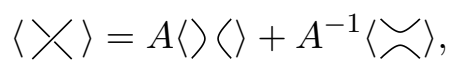


where $X$, ) ( and $\asymp$ stand for link diagrams which coincide outside a disk and in the disk look as their notations.

Let us categorify this skein relation. Consider the map

$$
\alpha: \mathcal{C}_{p, q}(\asymp) \rightarrow \mathcal{C}_{p, q-1}(\text { Х })
$$

which sends an enhanced state $S$ of $\asymp$ to the enhanced state of $久$ smoothing along which coincides with the smoothing of $\asymp$ along $S$ and the signs of the ovals are also the same. The collection of these maps is a chain homomorphism, that is, they commute with $d$. Indeed, the incidence numbers are the same for an enhanced Kauffman state of $\asymp$ and its images in $久$, and the latter cannot contain in the boundary a state with positive marker at $c$.

Now consider the map

$$
\left.\beta: \mathcal{C}_{p, q}(X) \rightarrow \mathcal{C}_{p, q-1}(\rangle\right)
$$

which sends each enhanced state with negative marker at $c$ to 0 and each enhanced state with positive marker at $c$ to the enhanced state of) (with the same smoothing and signs of the ovals. This is again a chain homomorphism.

The homomorphisms $\alpha$ and $\beta$ form a short exact sequence of complexes:

$$
0 \longrightarrow \mathcal{C}_{*, *}(\asymp) \stackrel{\alpha}{\longrightarrow} \mathcal{C}_{*, *-1}\left(\text { X) } \stackrel{\beta}{\longrightarrow} \mathcal{C}_{*, *-2}()() \longrightarrow 0\right.
$$

It induces a collection of long homology sequences:

$$
\begin{aligned}
& \stackrel{\partial}{\longrightarrow} H_{p, q}(\asymp) \stackrel{\alpha_{*}}{\longrightarrow} H_{p, q-1}\left(\text { Х) } \stackrel{\beta_{*}}{\longrightarrow} H_{p, q-2}(\rangle\right) \stackrel{\partial}{\longrightarrow} \\
& \stackrel{\partial}{\longrightarrow} H_{p-1, q}(\smile) \stackrel{\alpha_{*}}{\longrightarrow} H_{p-1, q-1}\left(\text { Х) } \stackrel{\beta_{*}}{\longrightarrow} H_{p-1, q-2}(\rangle\right) \stackrel{\partial}{\longrightarrow}
\end{aligned}
$$

A special case of this sequence, which relates the groups of a connected sum and disjoint sum of knots, can be found in Section 7.4 of Khovanov's paper [7]. This special case is the only one which can be formulated for the original version of homology depending on orientations of links.

The sequence (18) proved to be useful for calculation of Khovanov homology. For instance, it was used in [13] and [1].

I have not been able to categorify the Jones skein relation which involves the Jones polynomial of oriented links. The Jones skein relation can be deduced from a couple of Kauffman skein relations. However this algebraic manipulation seems to have no categorification.

\section{References}

[1] M. M. Asaeda and J. H. Przytycki, Khovanov homology: torsion and thickness, arXiv: math.GT/0402402. 
[2] D. Bar-Natan, Khovanov's categorification of the Jones polynomial, Algebr. Geom. Topol. 2 (2002), 337-370; arXiv: math.QA/0201043.

[3] - Khovanov's homology for tangles and cobordisms, arXiv: math.GT/0410495.

[4] M. Jacobsson, An invariant of link cobordisms from Khovanov's homology theory, arXiv: math.GT/0206303.

[5] I. B. Frenkel and M. Khovanov, Canonical bases in tensor products and graphical calculus for $U_{q}\left(\mathfrak{s l}_{2}\right)$, Duke Math. J. 87 (1997), 409-480.

[6] L. H. Kauffman, State models and the Jones polynomial, Topology 26 (1987), 395407.

[7] M. Khovanov, A categorification of the Jones polynomial, Duke Math. J. 101 (1999), 359-426; arXiv: math.QA/9908171.

[8] -, A functor-valued invariant of tangles, Algebr. Geom. Topol. 2 (2002), 665-741; arXiv: math.QA/0103190

[9] —, Patterns in knot cohomology I, Experimental Math. 12 (2003) 365-374; arXiv: math.QA/0201306.

[10] —, Categorifications of the colored Jones polynomial, Duke Math. J. 101 (2000), 359-426; arXiv: math.QA/0302060.

[11] —, sl(3) link homology I, arXiv: math.QA/0304375.

[12] M. Khovanov and L. Rozansky, Matrix factorization and link homology, arXiv: math.QA/0401268.

[13] E. S. Lee, The support of the Khovanov's invariants for alternating knots, arXiv: math.GT/0201105.

[14] —, On Khovanov invariant for alternating links, arXiv: math.GT/0210213.

[15] P. Ozsvath and Z. Szabo, Holomorphic disks and knot invariants, arXiv: math.GT/ 0209056.

[16] J. Rasmussen, Khovanov homology and the slice genus, arXiv: math.GT/0402131.

[17] A. Shumakovitch, Torsion of the Khovanov homology, arXiv: math.GT/0405474.

[18] O. Viro, Remarks on definition of Khovanov homology, arXiv: math.GT/0202199.

Department of Mathematics

Uppsala University

Box 480

S-751 06 Uppsala, Sweden

E-mail: oleg@math.uu.se

Received 20 May 2004;

in revised form 20 November 2004 